\title{
5,7-DHT-induced lesions of the nucleus basalis or frontal cortex do not block passive avoidance retention impairments produced by $p$-chloroamphetamine in rats
}

\author{
ANTHONY C. SANTUCCI, PETER J. KNOTT, and VAHRAM HAROUTUNIAN \\ Psychiatry Service, Bronx VAMC, Bronx, New York \\ and Department of Psychiatry, Mt. Sinai School of Medicine, New York, New York
}

\begin{abstract}
The effects of serotonergic lesions of the nucleus basalis of Meynert (nbM) or regions within the frontal cortex (FCTX) induced with 5,7-dihydroxytryptamine were examined to determine whether such damage would block the amnesia-producing effect of pretraining $(30 \mathrm{~min}$ ) $p$-chloroamphetamine (PCA; $2.5 \mathrm{mg} / \mathrm{kg}$ ) in rats trained on passive avoidance. Results indicated that, irrespective of their lesion condition, all groups treated with PCA exhibited 72-h retention impairments. Serotonergic lesions of the nbM or FCTX alone did not affect retention performance despite producing extensive depletions of serotonin (34.8\%-84.7\%) and 5-hydroxyindoleacetic acid (59.0\%-86.0\%). Neither lesion procedure, nor the administration of PCA, had an effect on activity levels of cholinergic markers choline acetyltransferase and acetylcholinesterase within the frontal cortex. These data demonstrate that the memory-impairing effects of PCA are not (1) dependent on the existence of releasable pools of serotonin in the nbM or FCTX, (2) mediated by stimulation of serotonergic receptors in the nbM or FCTX, or (3) related to disruption of cholinergic activity within the frontal cortex. These data also reveal that serotonergic lesions to the nbM or FCTX by themselves do not produce impairments in retention on passive avoidance.
\end{abstract}

Findings from a number of recent animal experiments have clearly indicated a role for serotonin in modulating learning and memory processes, though the exact nature of this modulation is not well understood. Many of these investigations have manipulated serotonergic neurotransmission by administering the releasing/depleting agent $p$-chloroamphetamine (PCA). Although a clear consensus regarding the effects of PCA on learning and memory has not been reached, a number of investigators have reported performance impairments when animals have been treated with PCA shortly prior to learning (Lalonde \& Vikis-Freibergs, 1985; Ögren, 1986a, 1986b; Ögren, Johansson, \& Magnusson, 1985; Riekkinen, Jäkälä, Sirviö, \& Riekkinen, 1991; Santucci, Kanof, \& Haroutunian, 1990; Santucci, Knott, \& Haroutunian, 1995).

Since PCA produces both acute increases (SandersBush \& Steranka, 1978) and long-term chronic depletions (Miller, Cox, Snodgrass, \& Maickel, 1970) of serotonin, it is important to assess which of these two biochemical effects contribute to the often reported retention impairment observed in PCA-treated animals. With passive

\footnotetext{
The contributions made by Renee Gluck and John Bangston to the present report are gratefully acknowledged. Support for this study was provided by an Alzheimer's Disease Research Center grant (P50AG05138) from the NIA. Correspondence concerning this article should be addressed to A. C. Santucci, who is now at the Department of Psychology, Manhattanville College, 2900 Purchase St., Purchase, NY 10577 (e-mail: santucci@mville.edu).
}

avoidance as the assessment instrument, we have obtained data indicating that PCA's effects on the release of serotonin account for deficits in retention (Santucci et al., 1995). Interestingly, results from this same study also revealed that PCA's effects on retention performance were related to increased serotonergic release from dorsal raphe cells, in that animals prepared with 5,7-dihydroxytryptamine neurotoxic lesions of the dorsal raphe nucleus were no longer susceptible to the amnestic properties of the drug. The fact that the effects of PCA could be ameliorated by preexisting lesions suggests that when the releasable pool of serotonin from the dorsal raphe is depleted, the amnesic properties of PCA are blocked.

In the present experiment, we explored further the anatomical bases for PCA's effects on learning and memory. Specifically, the present investigation determined whether the serotonergic projections to the nucleus basalis of Meynert (nbM) or to regions within the frontal cortex (FCTX) were involved in the mediation of PCA's effects on learning and memory. The nbM and FCTX were selected as the focus of study, because (1) cortically projecting cholinergic nbM and serotonergic dorsal raphe neurons overlap throughout the cortex (Bigl, Woolf, \& Butcher, 1982; Steinbusch, 1981), (2) both structures play critical roles in modulating learning and memory processes (Dekker, Connor, \& Thal, 1991; Haroutunian, Mantin, \& Kanof, 1990; Santucci, Kanof, \& Haroutunian, 1989; Wolf, Waksman, Finger, \& Almli, 1987), and (3) the dorsal raphe projects to the region of the nbM that 
is rich in serotonergic receptors (Pazos, Cortes, \& Palacios, 1985; Pazos \& Palacios, 1985). It was hypothesized that if either the release of serotonin from, or the stimulation of serotonergic receptors on, cells of the nbM or FCTX were important in mediating PCA's memory-impairing effects, then lesions to these structures would block PCA's amnestic effects.

\section{METHOD}

\section{Subjects}

Fifty-four male adult Sprague-Dawley rats purchased from Charles River (Wilmington, MA) were used. The animals were housed in groups of 3 or 4 in suspended wire-mesh cages $(40.6 \times$ $25.4 \times 17.8 \mathrm{~cm}$ ) and were maintained on a 12:12-h light:dark cycle with ad-lib access to Purina Rat Chow and water. Behavioral testing was conducted during the light phase between $10 \mathrm{a} . \mathrm{m}$. and 5 p.m.

\section{Apparatus}

Passive avoidance training and testing were conducted in a twocompartment black/white shuttlebox $(35 \times 28 \times 16 \mathrm{~cm})$ with a guillotine door separation and a stainless steel grid floor through which scrambled electric footshock could be administered.

\section{Procedure}

Drug preparations. The serotonergic neurotoxin 5,7-dihydroxytryptamine (5,7-DHT; Sigma Chemicals, St. Louis) was used as the infusant for all surgeries. The neurotoxin was mixed fresh daily with saline (SAL) and stored on ice in light-tight bottles. Ascorbic acid $(0.2 \mathrm{mg} / \mathrm{ml})$ was added to the 5,7-DHT solution to retard oxidation. Solutions containing $p$-chloroamphetamine HCL (PCA; Sigma Chemicals) were also mixed fresh daily with SAL and stored in light-tight bottles.

Surgeries. Subjects were prepared either with neurotoxic lesions of either the nbM or FCTX, or with sham lesions. Irrespective of its lesion condition, each subject was initially injected (i.p.) with $25-\mathrm{mg} / \mathrm{kg} / \mathrm{ml}$ desmethylimipramine (Sigma Chemicals) $20 \mathrm{~min}$ before administration of surgical anesthesia (Björklund, Baumgarten, \& Rensch, 1975). Following KETALAR $(60-\mathrm{mg} / \mathrm{kg} / \mathrm{ml} \mathrm{ke-}$ tamine HCL, i.m., Parke-Davis, Morris Plains, NJ) plus sodium pentobarbital $(21 \mathrm{mg} / \mathrm{kg} / \mathrm{ml}$, i.p., Sigma Chemicals) anesthesia, each rat was positioned in a Baltimore stereotaxic instrument with the UIB set at 0 . Bilateral lesions of the FCTX were achieved by infusing $1 \mu \mathrm{l}(15 \mu \mathrm{g} / 1 \mu \mathrm{l})$ of 5,7-DHT through a 33-g cannula at each of two hemispheric sites ( +3.5 from bregma, \pm 0.6 from midline sinus, and -4.0 and -3.0 from the skull). Neurotoxic lesions of the nbM were produced by infusing the neurotoxin bilaterally at stereotaxic coordinates -0.3 from bregma, \pm 3.0 from midline, and $-7.8(1.2 \mu \mathrm{l}),-7.5(0.4 \mu \mathrm{l})$, and $-7.2(0.4 \mu \mathrm{l})$ from the skull. The neurotoxin was infused slowly by hand over a period of several minutes. After the infusion was completed, the cannula remained in place for $1 \mathrm{~min}$ to allow for diffusion. Frontal cortical and $\mathrm{nbM}$ sham lesion procedures were identical to those described above, except that the cannula was lowered to a dorsal/ventral location above the lesion site and 5,7-DHT was not infused (FCTX dor$\mathrm{sal} / \mathrm{ventral}$ coordinate: -1.5 from skull; nbM dorsal/ventral coordinate: -6.0 from skull).

Passive avoidance. After a 2-week recovery period, subjects were trained on passive avoidance. Training consisted of placing an animal in the white compartment of the shuttlebox for $60 \mathrm{sec}$, after which time the guillotine door separating the two compartments was raised. Once the animal had crossed into the black compartment (typically within $30 \mathrm{sec}$ ), the guillotine door was lowered, and a mild, short-duration footshock $(1.0 \mathrm{~mA} / 2 \mathrm{sec})$ was administered. Subjects remained on the black side for 60 sec after shock termination. Thirty minutes prior to training, lesioned and sham animals were injected (i.p.) with either SAL or $2.5-\mathrm{mg} / \mathrm{kg}$ PCA.
The dose of PCA was based on previous work from our laboratory (Santucci et al., 1990; Santucci et al., 1995). Eight treatment conditions were thus formed: NBM/SHAM/SAL $(n=7)$, NBM/ SHAM/PCA $(n=6)$, NBM/5,7-DHT/SAL $(n=6)$, NBM/5,7DHT/PCA $(n=6)$, FCTX/SHAM/SAL $(n=7)$, FCTX/SHAM/ PCA $(n=7)$, FCTX/5,7-DHT/SAL $(n=7)$, and FCTX/5,7DHT/PCA $(n=8)$.

Retention testing, which was assessed $72 \mathrm{~h}$ after completion of training, was conducted in a manner identical to that of training, except that footshock was not administered. The latency to cross (with three paws) into the previously shocked black compartment served as the measure of retention. The apparatus was wiped clean with a deodorizer between testing of different animals.

Neurochemistry. Within approximately 2 weeks of completing passive avoidance testing, animals were sacrificed by rapid decapitation and their brains were dissected for neurochemical analysis. The brains from 5 animals were not included in the neurochemical assays because of death between end of testing and day of decapitation. Dissected tissue samples from the nbM and FCTX regions were obtained on dry ice in the following manner. First, each brain was placed in a stainless steel dissecting matrix, which allowed the dissector to make reliable $1-\mathrm{mm}$ thick coronal slabs. Ten such slabs were obtained beginning at the frontal poles and extending through the entire anterior-posterior extent of the nbM. Each coronal slab was placed on a microscope slide and dissected on a freezing dissecting table. Bilateral samples of the nbM were obtained by punching out a 1.2-mm-diameter area from the two slabs that most clearly contained the nbM region. Cortical tissue samples from animals with sham or 5,7-DHT lesions of the nbM were obtained from each coronal slab by dissecting off the cortical mantle superior to the rhinal fissure bilaterally. All cortical samples from each animal were combined and crushed into a fine powder. Two aliquots of the powdered tissue were made and used for assessment of aminergic and cholinergic markers. In animals with sham or 5,7DHT lesions of the FCTX, cortical samples were obtained as described above, except that the lesioned area (i.e., $1.5 \mathrm{~mm}$ anterior and $1.5 \mathrm{~mm}$ posterior to the cannula track) was kept separate from the remaining cortex and was not powdered. In these animals, the lesioned (unpowdered) cortical sample was used to assess amines while the remaining (powdered) cortex was used to measure cholinergic markers.

Lesion efficacy was established by assessing concentrations of serotonin (5-HT) and 5-hydroyindoleacidic acid (5-HIAA) within the nbM and FCTX in animals that served in the NBM/5,7-DHT/ SAL and FCTX/5,7-DHT/SAL groups. Values obtained were then compared with control values derived from the NBM/SHAM/SAL and FCTX/SHAM/SAL conditions, respectively (see Table 1). Concentrations of 5-HT and 5-HIAA were measured by HPLC-EC, using a modification of the methods of Maruyama, Oshima, and Nakajima (1980). Activity levels of choline acetyltransferase (CAT) and acetylcholinesterase (AChE) within the FCTX were measured according to the respective methods of Fonnum (1975) and Johnson and Russel (1975). These measurements were performed to eliminate the possibility that perturbations to the nbM-frontal cortical cholinergic system contributed to performance impairments.

\section{RESULTS}

\section{Passive Avoidance}

The mean retention latencies for the eight groups of subjects are presented in Figure 1. Retention scores were analyzed with a 2 (region) $\times 2$ (lesion) $\times 2$ (PCA) completely randomized analysis of variance (ANOVA). The results revealed a main effect only of PCA $[F(1,46)=$ $40.0, p<.001]$, with PCA-treated animals exhibiting lower retention latencies than those of SAL controls. No 
Table 1

Mean ( \pm SEM) 5-HT and 5-HIAA Concentrations

$(\mu \mathrm{g} / \mathrm{g}$ wet tissue) Within the FCTX and nbM

in 5,7-DHT and Sham-Operated Animals

\begin{tabular}{|c|c|c|c|c|c|c|c|c|}
\hline \multirow[b]{3}{*}{ Group } & \multicolumn{4}{|c|}{ FCTX } & \multicolumn{4}{|c|}{ nbM } \\
\hline & \multicolumn{2}{|c|}{$5-\mathrm{HT}$} & \multicolumn{2}{|c|}{ 5-HIAA } & \multicolumn{2}{|c|}{ 5-HT } & \multicolumn{2}{|c|}{ 5-HIAA } \\
\hline & $M$ & $S E M$ & $M$ & $S E M$ & $M$ & $S E M$ & $M$ & $S E M$ \\
\hline \multicolumn{9}{|l|}{ nbM Groups } \\
\hline NBM/SHAM/SAL & 0.189 & .032 & 0.125 & .027 & 0.209 & .041 & 0.221 & .031 \\
\hline $\mathrm{NBM} / 5,7-\mathrm{DHT} / \mathrm{SAL}$ & 0.068 & .034 & 0.025 & .003 & 0.032 & .016 & 0.031 & .013 \\
\hline \multicolumn{9}{|l|}{ FCTX Groups } \\
\hline FCTX/SHAM/SAL & 0.155 & .026 & 0.156 & .028 & 0.103 & .040 & 0.123 & .032 \\
\hline FCTX/5,7-DHT/SAL & 0.101 & .019 & 0.064 & .013 & 0.168 & .059 & 0.151 & .047 \\
\hline
\end{tabular}

other main effect or interaction reached statistical significance (all $p s>.20$ ). The lower retention latencies of PCA-treated animals were not related to a drug-induced increase in locomotion; SAL- and PCA-treated rats entered the black compartment at training with an equal propensity $(p>.20)$.

\section{Neurochemistry}

Mean 5-HT and 5-HIAA concentrations within the $\mathrm{nbM}$ and FCTX for the NBM/SHAM/SAL, NBM/5,7DHT/SAL, FCTX/SHAM/SAL and FCTX/5,7-DHT/SAL groups are presented in Table 1.

Separate 2 (region) $\times 2$ (lesion) completely randomized ANOVAs were used to analyze the data set for each neurochemical within each brain area. Serotonergic lesions of the nbM or FCTX produced significant depletions of 5-HT and 5-HIAA within the FCTX [main effects of lesion: both $F \mathrm{~s}(1,20)>8.40$, both $p \mathrm{~s}<.01$ ]. In contrast, depletions of serotonergic markers within the nbM were observed only in animals prepared with 5,7DHT lesions of the $\mathrm{nbM}$ [region $\times$ lesion interactions: both $F s(1,20)>5.94$, both $p s<.05$ ].

As can be seen in Table 2, neither lesions of the nbM or FCTX nor administration of PCA affected cortical CAT and $\mathrm{AChE}$ activity levels [all $F_{\mathrm{S}}(1,38)<2.29$, all $p \mathrm{~s}>.10$ ]

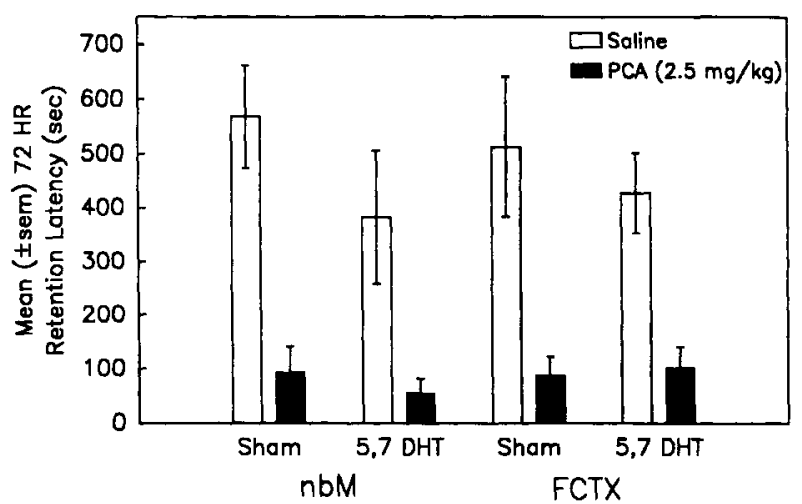

Figure 1. Mean $( \pm S E M)$ 72-h retention latencies for the eight groups of subjects. Irrespective of their lesion status, all groups treated with PCA 30 min prior to passive avoidance training exhibited impaired retention scores. Serotonergic 5,7-DHT lesions of the nbM or FCTX alone were without effect on retention performance.

\section{DISCUSSION}

The present data indicated that serotonergic lesions of either the nbM or FCTX did not block the amnesiaproducing effect of pretraining PCA. Moreover, since cortical cholinergic markers were unaltered when measured within 2 weeks of behavioral testing, the present findings also demonstrated that PCA's effect on memory was independent of cholinergic changes within the frontal cortex. These results suggest that serotonergic release from, or stimulation of serotonergic receptors on, cells of the nbM or FCTX do not contribute to PCA's memory impairing effect. The failure of these lesions to interfere with PCA's influence on memory processes occurred despite the effectiveness of the lesions in depleting serotonin and its major metabolite. These data, together with recently obtained findings indicating an important role for the dorsal raphe nucleus in mediating PCA's effect (Santucci et al., 1995), suggest that although serotonergic systems are involved in the learning and memory deficits produced by PCA, these effects must be mediated by anatomic sites other than the nbM or FCTX.

A more mundane noncognitive alternative interpretation of the present data would suggest that pretraining PCA administration simply interfered with the animals' ability to sense the training footshock. Although no formal assessment of this possibility was made in the present study, previous data from our laboratory has suggested that, at this dose, PCA does not affect footshock threshold levels (Santucci et al., 1995). Indeed, the consensus derived from a review of the literature regarding the role of serotonin in pain has indicated that PCA does not affect the sensitivity of experimental animals to detect nociceptive stimuli (Le Bars, 1988). In fact, the author of this review noted that in one study PCA produced a decrease in pain threshold (Le Bars, 1988). Therefore, in the present study, an increase in pain thresholds most likely did not account for the poor performance exhibited by PCA-treated animals.

Caution, however, is needed when concluding that neither the FCTX nor the nbM plays a critical role in mediating PCA's effect. More complete lesions, or lesions to other subpopulations of serotonergic cells which project to these brain structures, might be necessary before a blockade of PCA's effect can be realized. This is especially 
Table 2

Mean ( \pm SEM) CAT (nmol ACh/hr/mg Protein) and AChE (nmol AChE/hr/mg Protein) Activities Within the FCTX in 5,7-DHT and Sham-Operated Animals Treated With Either SAL or PCA $(2.5 \mathrm{mg} / \mathrm{kg}) 30 \mathrm{~min}$ Prior to Training

\begin{tabular}{lrrrrr}
\hline & \multicolumn{2}{c}{ CAT } & & \multicolumn{2}{c}{ AChE } \\
\cline { 5 - 6 } \cline { 5 - 6 } Group & $M$ & $S E M$ & & SEM \\
\hline nbM Groups & & & & \\
NBM/SHAM/SAL & 25.8 & 0.91 & 1949 & 45.8 \\
NBM/SHAM/PCA & 23.7 & 1.68 & 2002 & 72.5 \\
NBM/5,7-DHT/SAL & 26.2 & 4.33 & 1873 & 246.1 \\
NBM/5,7-DHT/PCA & 22.9 & 1.41 & 1818 & 65.7 \\
FCTX Groups & & & & \\
FCTX/SHAM/SAL & 26.4 & 1.02 & 2153 & 82.0 \\
FCTX/SHAM/PCA & 27.8 & 1.09 & 2072 & 80.8 \\
FCTX/5,7-DHT/SAL & 25.6 & 0.79 & 2012 & 67.7 \\
FCTX/5,7-DHT/PCA & 26.5 & 1.08 & 2082 & 73.6 \\
\hline
\end{tabular}

true with respect to the frontal cortex. Only a relatively small region of the frontal cortex was damaged in the present study. Although there exist data indicating the relevance of this particular area to learning and memory (Haroutunian et al., 1990; Santucci et al., 1989; Santucci, Kanof, \& Haroutunian, 1991; Welner, Dunnett, MacLean, \& Iversen, 1988; Wolf et al., 1987), other frontal cortical sites need to be examined before one can satisfactorily eliminate a role for the frontal cortex in this phenomenon.

Apart from addressing issues related to identifying the anatomical basis for PCA's effect, the present study is relevant in yet another way. Serotonergic lesions of the nbM or FCTX were found by themselves to be without effect on retention of passive avoidance. This finding contrasts with other literature indicating that cholinergic activities related to these brain regions are important for learning and memory. For example, many studies have consistently demonstrated performance impairments on tests of learning and memory in animals prepared with cholinergic lesions of the nbM (see Dekker et al., 1991, for a review). In addition, transplants of cholinergically rich cells into the frontal cortex have reversed the memory deficits in nbM-lesioned animals (Santucci et al., 1991; Welner et al., 1988). Finally, a region of the frontal cortex similar to the one examined here was found to mediate physostigmine's memory-enhancing effect in animals prepared with nbM lesions (Haroutunian et al., 1990). Taken together, these findings suggest the existence of some degree of neurochemical specificity. That is, since cholinergic, but not serotonergic, manipulations are effective, it appears that simply manipulating any neurochemical system of the nbM or FCTX is not sufficient to affect learning and memory.

In conclusion, the present results indicate that PCA's amnesia-producing effect is not related to serotonergic activity involving frontal cortical or nbM cells. Other studies examining more expansive regions or other subpopulations of cells, however, are needed before one can accept this interpretation fully.

\section{REFERENCES}

Bigl, V., Woolf, N. J., \& Butcher, L. L. (1982). Cholinergic projections from the basal forebrain to frontal, parietal, temporal, occipital, and cingulate cortices: A combined fluorescent tracer and acetylcholinesterase analysis. Brain Research Bulletin, 8, 727-749.

Björklund, A., Baumgarten, H. G., \& Rensch, A. (1975). 5,7Dihydroxytryptamine: Improvement of its selectivity for serotonin neurons in the CNS by pretreatment with desipramine. Journal of Neurochemistry, 24, 833-835.

DekKer, A. J. A. M., Connor, D. J., \& Thal, L. J. (1991). The role of cholinergic projections from the nucleus basalis in memory. Neuroscience \& Biobehavioral Reviews, 15, 299-317.

FonNuM, F. (1975). A rapid radiochemical method for the detection of choline acetyltransferase. Journal of Neurochemistry, 24, 407-408.

Haroutunian, V., Mantin, R., \& Kanof, P. D. (1990). Frontal cortex as the site of action of physostigmine in nbM-lesioned rats. Physiology \& Behavior, 47, 203-206.

JOHNSON, C. D., \& Russel, R. L. (1975). A rapid, simple radiometric assay for acetylcholinesterase, suitable for multiple determinations. Annals of Biochemistry, 26, 229-232.

LALONDE, R., \& VIKIS-Freibergs, V. (1985). Manipulations of 5-HT activity and memory in the rat. Pharmacology, Biochemistry \& Behavior, 22, 377-382.

LE BARS, D. (1988). Serotonin and pain. In N. N. Osborne \& M. Hamon (Eds.), Neuronal serotonin (pp. 171-229). New York: Wiley.

MaruYama, Y., Oshima, T., \& NaKaJima, E. (1980). III. Simultaneous determination of catecholamines in rat brain by reversed-phase liquid chromatography with electrochemical detection. Life Sciences, 26, 1115-1120.

Miller, F. P., CoX, R. H., SNodgrass, W. R., \& Maickel, R. P. (1970). Comparative effects of $p$-chlorophenylalanine, $p$-chloroamphetamine and $p$-chloro- $N$-methylamphetamine on rat brain norepinephrine, serotonin and 5-hydroxyindole-3-acetic acid. Biochemical Pharmacology, 19, 435-442.

ÖGREN, S. O. (1986a). Analysis of the avoidance learning deficit induced by the serotonin releasing compound $p$-chloroamphetamine. Brain Research Bulletin, 16, 645-660.

ÖGREN, S. O. (1986b). Serotonin receptor involvement in the avoidance learning deficit caused by $p$-chloroamphetamine-induced serotonin release. Acta Physiologica Scandinavica, 126, 449-462.

Ögren, S. O., Johansson, C., \& Magnusson, O. (1985). Forebrain serotonergic involvement in avoidance learning. Neuroscience Letters, 58, 305-309.

Pazos, A., Cortes, R., \& PAlacios, J. M. (1985). Quantitative autoradiographic mapping of serotonin receptors in the rat brain: II. Serotonin-2 receptors. Brain Research, 346, 231-249.

Pazos, A., \& Palacios, J. M. (1985). Quantitative autoradiographic mapping of serotonin receptors in the rat brain: I. Serotonin-1 receptors. Brain Research, 346, 205-230.

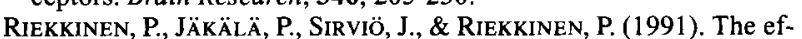
fect of increased serotonergic and decreased cholinergic activities on spatial navigation performance in rats. Pharmacology, Biochemistry \& Behavior, 39, 25-29.

Sanders-Bush, E., \& Steranka, L. A. (1978). Immediate and longterm effect of $p$-chloroamphetamine on brain amines. In J. H. Jacoby \& L. D. Lytle (Eds.), Serotonin neurotoxins (Annals of the New York Academy of Sciences, Vol. 305, pp. 208-221). New York: New York Academy of Sciences.

Santucci, A. C., Kanof, P. D., \& Haroutunian, V. (1989). Infusion of NMDA into the nucleus basalis of Meynert, frontal cortex or lateral ventricle in rats: Effect on memory and cholinergic brain neurochemistry. Pharmacology, Biochemistry \& Behavior, 33, 485488.

Santucci, A. C., Kanof, P. D., \& Haroutunian, V. (1990). Serotonergic modulation of cholinergic systems involved in learning and memory in rats. Dementia, 1, 151-155.

Santucci, A. C., Kanof, P. D., \& Haroutunian, V. (1991). Fetal transplant-induced restoration of spatial memory in rats with lesions 
of the nucleus basalis of Meynert. Journal of Neural Transplantation \& Plasticity, 2, 65-74.

Santucci, A. C., Knott, P. J., \& Haroutunian, V. (1995). Excessive serotonergic release, not depletion, leads to memory impairments in rats. Manuscript submitted for publication.

STEINBUSCH, H. W. M. (1981). Distribution of serotonin-immunoreactivity in the central nervous system of the rat-cell bodies and terminals. Neuroscience, 6, 557-618.

Welner, S. A., DunNetT, S. B., Maclean, B., \& Iversen, S. D. (1988).

Transplantation of embryonic ventral forebrain grafts to the neo- cortex of rats with bilateral lesions of nucleus basalis magnocellularis ameliorates a lesion-induced deficit in spatial memory. Brain Research, 463, 192-197.

Wolf, C., WAKSMAN, D., Finger, S., \& AlMLI, C. R. (1987). Large and small medial frontal cortex lesions and spatial performance of the rat. Brain Research Bulletin, 18, 1-5.

(Manuscript received August 29, 1994; revision accepted for publication January 10, 1995.) 